\title{
Dynamic cardiac PET imaging: Technological improvements advancing future cardiac health
}

\author{
Grant T. Gullberg, PhD, ${ }^{\mathrm{a}}$ Uttam M. Shrestha, $\mathrm{PhD},{ }^{\mathrm{a}}$ and Youngho Seo, $\mathrm{PhD}^{\mathrm{a}}$ \\ a Department of Radiology and Biomedical Imaging, University of California, San Francisco, CA
}

Received Jan 12, 2018; accepted Jan 15, 2018

doi: $10.1007 / \mathrm{s} 12350-018-1201-3$

\section{See related article, pp. 1286-1291}

While PET, in conjunction with $\mathrm{CT}^{1}$, has been an important tool in the management of oncology patients, accounting for $86 \%$ of PET scans by $2016,{ }^{2}$ PET with attenuation and motion correction has significant potential for future cardiac applications, especially with the ability of PET to perform dynamic imaging to measure myocardial perfusion-myocardial blood flow (MBF) and coronary flow reserve (CFR), integrity of neural transmitters of the autonomic nervous system, and connecting cardiac efficiency with metabolism of myocardial substrates. Its potential is found with its excellent resolution and sensitivity, the ability to use tracers with a short half-life allowing higher doses, and possibly in the future the ability to use ${ }^{18} \mathrm{~F}$ perfusion agents that would eliminate the need of an onsite cyclotron. During dynamic imaging (a dynamic scan should be performed for every procedure), the high counts during the blood input phase can paralyze the camera electronics. Therefore, improved time-of-flight (TOF) electronics able to accept these high counts rates and quality control measures to determine the maximum allowable injected dose (as presented in this issue of the Journal by van Dijk and colleagues) for dynamic cardiac PET studies need to be implemented. However, there is a caveat: restricting the injected dose to meet the count rate capabilities during the input phase penalizes the ability to obtain high counts during the later phase of the dynamic study when the camera electronics are less likely to be paralyzed. Using constant infusion

Reprint requests: Grant T. Gullberg, PhD, Department of Radiology and Biomedical Imaging, University of California, 185 Berry St., Ste 350, San Francisco, CA 94143-0946; gtgullberg@lbl.gov J Nucl Cardiol 2019;26:1292-7.

1071-3581/\$34.00

Copyright (C) 2018 American Society of Nuclear Cardiology. techniques can reduce high peak counts during the input phase, but this reduces the ability to accurately measure the frequency response of the transfer of blood to tissue compartments. Other approaches in the future such as using a library of input functions or blind estimation may alleviate the limitations of injecting a restricted dose for dynamic cardiac PET studies. Nevertheless, one needs to follow caution and consider the fact that the overall radiation burden to the U.S. population doubled from the early 1980 s to 2006, and the contribution of nuclear cardiology procedures to ionizing radiation burden increased 10 -fold. ${ }^{3}$ Thus new and improved hardware, software, and radiotracer developments play an important part in the performance of the PET system for dynamic cardiac applications to provide expected clinical benefit that outweighs the risks of the procedure.

In the paper by van Dijk and colleagues a simple method, originally proposed by Renaud et al., ${ }^{4}$ was used to determine the maximum activity of ${ }^{82} \mathrm{Rb}$ allowed for estimating MBF using the new Philips digital PET camera with time-of-flight capability. The method is similar to that of an earlier method used by our group at UCSF for determining the maximum activity of ${ }^{18} \mathrm{~F}$ tracers allowed for performing kinetic analysis via image derived blood input measurements from the heart using the Siemens Biograph 16 PET/CT. ${ }^{5}$ Basically, the method injects high levels of radioactivity into a phantom such as a cardiac insert of an anthropomorphic torso phantom (Data Spectrum Corp) that saturates the system. With time, the activity decays decreasing the dead time or paralysis in the system allowing the counts to increase (see illustration in Figure 1). When the count rate increases to a level where the bias between the true activity and the measured activity is less than some level of accuracy, such as $1 \%$, that determines the activitymodified for patient size and weight - that one can inject to obtain accurate kinetic parameters for the estimation of myocardial blood flow (MBF).

In the work of van Dijk and colleagues, data were acquired on three LYSO TOF PET systems: Two analog 
A

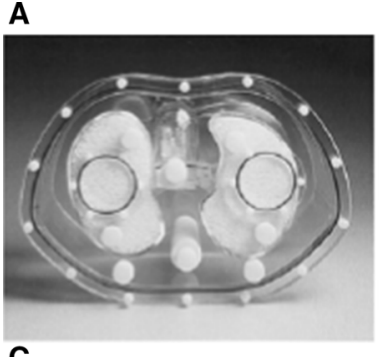

B

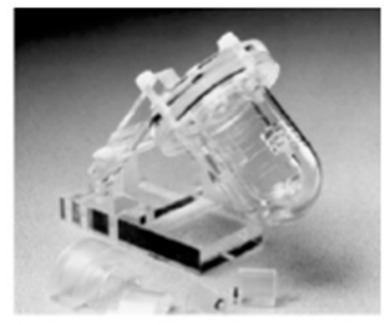

C
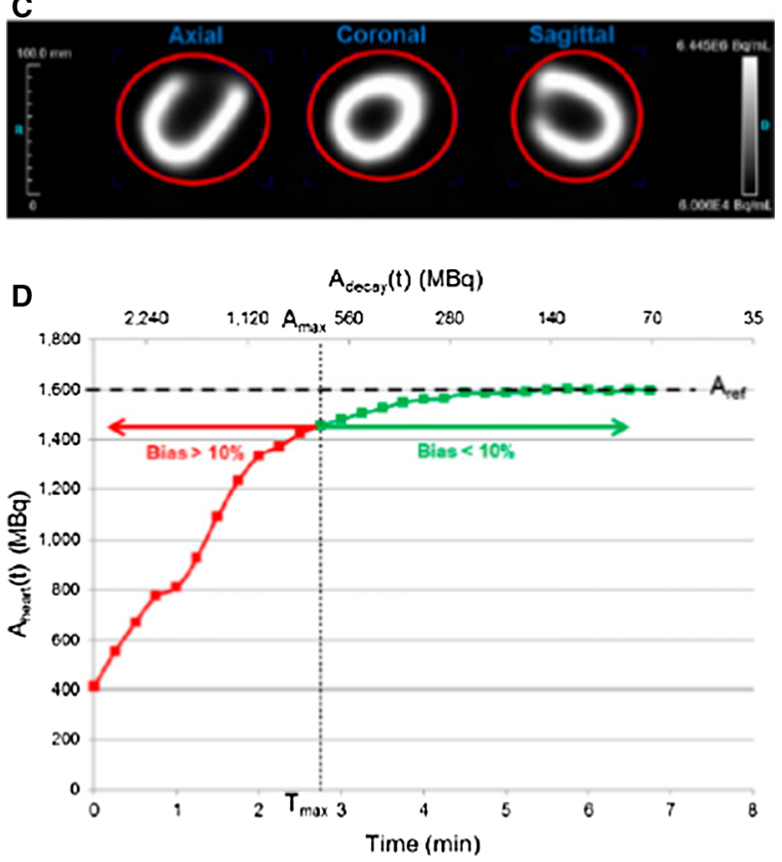

Figure 1. Figure in the paper of Renaud and colleagues ${ }^{4}$ showing the anthropomorphic torso phantom used for the measurements (A) with cardiac insert (B). The volume of interest (red) (C) is drawn over the cardiac insert. An illustration of the acquired data shows the measured timeactivity curve (D) increasing with time as the paralysis of the system deceases with a decrease in activity due to decay. The measured data approaches asymptotically the true activity. Dotted (vertical) line shows the time when the accurate and inaccurate measured activity is less than $1 \%$. This figure was originally published in Journal of Nuclear Medicine. ${ }^{4}$ () by the Society of Nuclear Medicine and Molecular Imaging, Inc.

systems-Ingenuity TF (Philips Healthcare) ${ }^{6}$ and Discovery 690 (GE Healthcare) ${ }^{7}$ - were compared with the new digital PET prototype camera from Philips Healthcare. ${ }^{8}$ Data from these three systems were reconstructed into 40 dynamic images, each of $15 \mathrm{~s}$, using vendor iterative expectation maximization reconstruction software with corrections for attenuation, scatter, randoms, detector efficiency, dead time, and decay. The phantom results showed that the three scanners were limited to a maximum injection of 312 $\mathrm{MBq}(8.43 \mathrm{mCi})$ for the Philips Ingenuity TF, $650 \mathrm{MBq}$
(17.57 $\mathrm{mCi}$ ) for the GE Discovery 690, and $654 \mathrm{MBq}$ $(17.67 \mathrm{mCi})$ for the Philips digital PET prototype to insure that the reconstructed images would not deviate more than $1 \%$ from the true activity in a dynamic cardiac study. It is interesting that the analog GE D690 system with a timing resolution (TR) of 544.3 picoseconds (ps) compared well with the Philips digital PET prototype (TR $=307 \mathrm{ps)}$ and much better than the Ingenuity $\mathrm{TF}(\mathrm{TR}=502 \mathrm{ps})$. For a clinical study a physician needs to know how these results translate for a particular patient whose body habitus can change dramatically. This is discussed in the paper of van Dijk and colleagues by anticipating patient-injected doses using conversion results in the literature for other PET systems. However, they emphasize that these studies still need to be performed for the Philips digital PET system. An important further study is to evaluate the effects of these results on the accuracy and precision of the estimated nonlinear kinetic parameters, which depend significantly upon the data statistics and the algorithmic methods used. These measurements have important implications for using PET to measure MBF and for other applications requiring dynamic imaging to measure coronary flow reserve, pre- and post-synaptic neuronal transmission rates, cardiac metabolic rates, and cardiac efficiency. Rubidium-82 has a relatively short half-life of 76s and high activities are therefore required to obtain sufficient image quality; whereas ${ }^{18} \mathrm{~F}$-agents would expect to provide overall better accuracy and precision because the longer half-life of 110 minutes allows for better statistics during the wash-out phase of the kinetic analysis.

PET has made substantial hardware improvements advancing TOF capability over the last 40 years making significant impact upon image quality. ${ }^{9}$ The idea to use TOF information was originally proposed early on in the development of PET, and the first TOF PET systems were industrialized in the 1980s. ${ }^{10}$ A good review of these early developments ${ }^{11}$ revealed several hardware deficiencies at that time. For one, dead time due to event pileup was a very significant problem limiting TOF systems to operate only in 2D mode, but the new technology has allowed modern PET systems to operate TOF in 3D mode. Major hardware advancements include new detector materials, such as Lutetium (Yttrium) Oxyorthosilicate (LSO and LYSO), providing excellent stopping power for $511 \mathrm{keV}$ photons with high photon yield at fast rise times; more compact $\mathrm{SiPM}^{12}$ compared to PMTs, with faster photon amplification, capable of being inserted into a MRI scanner; accessibility of application-specific integrated circuits (ASICs) allowing more dense and complex electronics; and faster computing hardware to process data. TOF imaging thought not possible in the early scanners developed in 
the 80s is now possible and has significant advantages. The accuracy of photon detection, the uniformity across the detector, and the TOF resolution have been improved by eliminating photomultipliers and Anger logic and using SiPMs with 1:1 coupling. Additional improvements come from improving crystal depth of interaction, energy resolution, and timing estimation in PET detectors with deconvolution and maximum likelihood pulse shape discrimination. ${ }^{13}$ It may be possible that in the future PET systems will have coincidence timing resolutions of 10 ps providing a resolution of 5 $\mathrm{mm}$ without requiring any reconstruction; however, in order to reach the 10 ps range radically new approaches must be developed for scintillator light generation, light transport, and light conversion. ${ }^{14}$ One step in this direction is to have fully digital PET detectors without photon conversion to visible light ${ }^{15}$ such as in cadmium zinc telluride detectors presently being used in some SPECT systems. Another promising development is using a library of known waveforms to train deep convolutional neural networks (CNNs) to estimate PET time-of-flight from the leading edge of a coincident waveform. Berg and Cherry have shown that this improves coincidence timing by as much as $23 \% .{ }^{16}$ In the future, CNNs could be used to improve sampling rate, resolution, partial volume, and noise in the waveform due to electronic and statistical noise.

The development of PET reconstruction algorithms has also had an important impact on the ability to process cardiac PET data and to accurately model the physical effects of the imaging detection process. ${ }^{17,18}$ Advanced algorithms have significantly improved image quality with corrections for physical effects of attenuation, detector efficiency, detector geometric response, scatter, randoms, prompt gammas, dead time, and changes in TOF resolution as a function of measured detector count rate. ${ }^{19}$ Acquisition of list-mode data temporally marks every event according to TOF position in the acquired data. Anatomical positions of heart tissue during the cardiac and respiratory cycle can be sorted by these timing marks for which TOF list-mode iterative maximum likelihood algorithms with correction for motion can accurately reconstruct the position of events, significantly improving cardiac image quality. With the aim of reducing dose, adaptive statistical iterative reconstruction algorithms can also be used to reconstruct CT scans of low dose for attenuation correction. ${ }^{20}$ Furthermore, the addition of TOF capability presents some very interesting mathematical subtleties from which one can infer attenuation ${ }^{21}$ without requiring a $\mathrm{CT}$ scan, and can also record motion. ${ }^{22}$ With the advancement in deep learning it is conceivable that, in the future, CNN might be used to prescribe count levels during a brief time of high counts and thus maintain accurate high counts during the other time of the scan when the count level does not saturate the camera. Thus, both advanced hardware and algorithms that accurately model physical effects of the image detection process are key for the estimation of accurate parametric images ${ }^{23}$ of physiological processes that provide clinical parameters of $\mathrm{MBF}, \mathrm{CFR}$, synaptic neuronal uptake and wash-out, and metabolic rates from dynamic data.

Radionuclide myocardial perfusion imaging (MPI) is the most established cardiovascular imaging technique with significant clinical benefits including diagnosing the presence and extent of coronary artery disease (CAD), estimating functional importance of hemodynamically significant epicardial disease, and improving risk stratification of patients. ${ }^{24}$ MPI PET tracers that are FDA approved and reimbursable are limited to the generator produced ${ }^{82} \mathrm{Rb}$ (75 seconds) and cyclotron produced ${ }^{13} \mathrm{NH}_{3}$ (9.96 minutes). $\mathrm{H}_{2}^{15} \mathrm{O}$ (122 seconds) is the ideal perfusion tracer but its fast decay makes it difficult to handle in routine clinical operations and its poor contrast is not ideal for lesion detection. ${ }^{18} \mathrm{~F}$-tagged agents (110 minutes) would be preferred due to advantages of better positron range than ${ }^{82} \mathrm{Rb}$ and not requiring a cyclotron on site. The ${ }^{18} \mathrm{~F}$-labeled Flurpiridaz is currently in clinical trials, is an inhibitor of mitochondrial complex I (MC-I), has a high first-pass extraction with slow wash out, has demonstrated an excellent relationship to flow in animal models, and has shown good diagnostic accuracy for the detection of CAD in humans. ${ }^{25} \mathrm{We}$ found in animal studies that ${ }^{18} \mathrm{~F}$ fluorodihydrorotenol ( $\left.{ }^{18} \mathrm{FDHROL}\right)$, an analog of rotenone, which also targets MC-I, provided better contrast than ${ }^{13} \mathrm{NH}_{3}$ (Figure 2). We also found that the wash-in rate constant of ${ }^{18}$ FDHROL in the hypertensive spontaneous hypertensive rat (SHR) was higher throughout its life than the normotensive WKY, ${ }^{26}$ likely due to increased oxygen needs of the hypertrophied myocytes where enlarged muscle fibers increase diffusion distances making it far less efficient. ${ }^{27}$

PET is ideal for researching and diagnosing the autonomic nervous system because of the ability to image the numerous ${ }^{11} \mathrm{C}$-tagged pre- and post-synaptic radiotracers, developed over the last 25 years, capable of distinguishing subcellular catecholamine dysfunction from complete denervation of cardiac sympathetic and parasympathetic innervation. ${ }^{28,29}$ The FDA has approved the SPECT tracer ${ }^{123}$ I-MIBG for evaluation of those patients with Class II-III congestive heart failure (CHF) and $\mathrm{LVEF}<35 \% .{ }^{30} \mathrm{MIBG}$ is an analog of the false neurotransmitter guanethidine taken up by adrenergic neurons of the cardiac sympathetic nervous system, similar to norepinephrine and allows a unique characterization of alterations in cardiac regional sympathetic nerve function. Using ${ }^{123} \mathrm{I}-\mathrm{MIBG}$ and ${ }^{201} \mathrm{Tl}$ with 

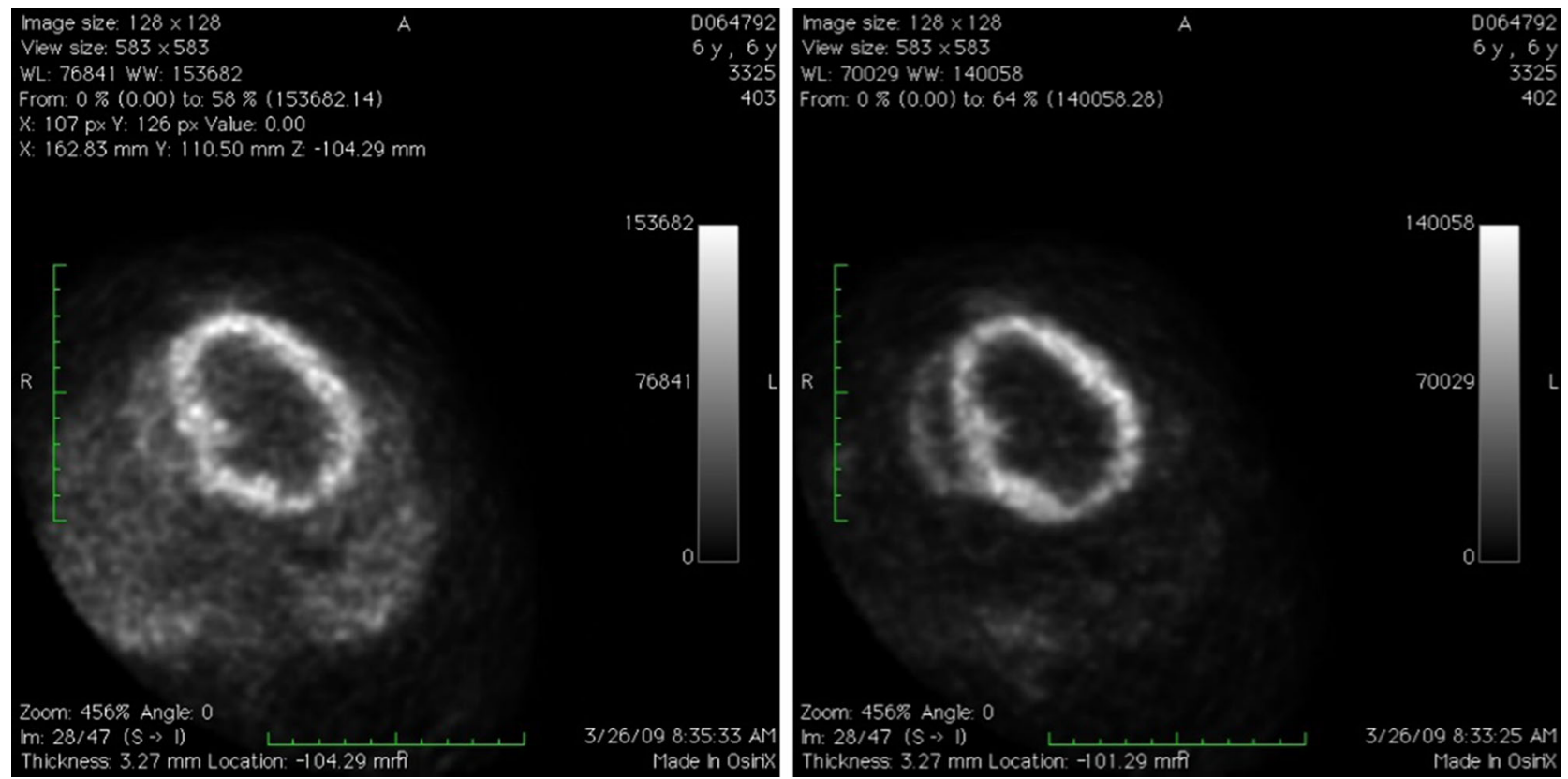

Figure 2. Cardiac PET images of a pig heart using the GE Discovery VCT PET/CT (GE Healthcare). Image on the left was acquired for $15 \mathrm{~min}$ with $906.5 \mathrm{MBq}(24.5 \mathrm{mCi})$ of ${ }^{13} \mathrm{NH}_{3}$ and the image on the right was acquired for $25 \mathrm{~min}$ with $888 \mathrm{MBq}(24 \mathrm{mCi})$ of ${ }^{18} \mathrm{~F}$-fluorodihydrorotenol $\left({ }^{18} \mathrm{FDHROL}\right)$. The time between the end of the ${ }^{13} \mathrm{NH}_{3}$ scan and the start of the ${ }^{18} \mathrm{FDHROL}$ scan was $40 \mathrm{~min}$. Notice the visualization of the right ventricle with ${ }^{18}$ FDHROL.

dynamic pinhole SPECT, we found in our study of the spontaneous hypertensive rat (SHR) that the abnormal nervous system activity in hypertrophic cardiomyopathy appears earlier than perfusion ${ }^{31}$ and may be indicative of a failing heart in late stages of heart failure (HF). Comparable results using the analogous PET agent ${ }^{11} \mathrm{C}$ meta-hydroxyephedrine $\left({ }^{11} \mathrm{C}\right.$-HED) have been reported, where PET imaging in $\mathrm{HF}$ patients with preserved ejection fraction (HFpEF) has been able to predict lethal arrhythmias, sudden cardiac death, response to cardiac resynchronization therapy, and relate severity of diastolic dysfunction to impaired cardiac sympathetic nervous system innervation. ${ }^{28,29}$ The longer half-life tracers, such as ${ }^{18} \mathrm{~F}$-LMI1195, has similar tracer kinetics to ${ }^{123} \mathrm{I}-\mathrm{MIBG}$ and would be a preferred PET radiotracer to image the autonomic nervous system. ${ }^{32}$ The use of PET to image the cardiac parasympathetic nervous system radiotracers is limited; however, ${ }^{11} \mathrm{C}$-donepezil a reversible antagonist of acetylcholine, has shown potential efficacy in initial studies. ${ }^{29}$

Dynamic PET imaging of glucose metabolism, fatty acid metabolism, and oxygen utilization provides information about metabolic shifts related to function, ${ }^{33}$ making PET an outstanding tool in conjunction with MRI for measuring cardiac efficiency. ${ }^{34}$ The three main metabolic substrates of the heart are carbohydrates, fatty acids, and ketone bodies with exogenous fatty acids accounting for $60 \%-70 \%$ of the energy production in the normal heart. ${ }^{35}$ In health, the myocardium increases fatty acid metabolism with an acute reduction in mechanical function, ${ }^{36}$ but conversely acute reduction in myocardial fatty acid metabolism in cardiomyopathy is also associated with impaired function. ${ }^{37}$ In the case of pressure overload, we found in studies using the SHR an increased reliance on carbohydrate oxidation in an attempt to maintain contractile function with a decline in fatty acid and oxygen utilization. ${ }^{26}$ Additionally, in PET/MRI studies of the normal Lewis rat, we found that glucose metabolic rate was inversely proportional to tissue work ${ }^{38}$ indicating that a more efficient metabolic substrate such as fatty acid would more likely be proportional to increased work. These results are exciting and provide an impetuous to study cardiac efficiency in relation to glucose and fatty acid metabolism in health and disease. Using PET/MRI one can measure myocardial efficiency on a tissue-by-tissue region of interest by calculating the ratio of external cardiac tissue work using MRI to oxygen utilization using PET measures of ${ }^{11} \mathrm{C}$-acetate kinetics as a surrogate for energy input. ${ }^{34}$ Work (work $=$ force $\times$ distance) is obtained by MRI measures of distance (strain) and force (stress), inferred from the inclusion of intraventricular pressure measurements in a finite element (FE) electromechanical cardiac model. 
Dynamic cardiac PET will make significant inroads in the future in the management of patients with cardiac disease. PET is ideal for performing perfusion, innervation, and metabolic studies. The combination of using PET to study metabolism with MRI to study work allows one to study the efficiency of the heart in disease. With the progress made over the last 40 years, it is conceivable that a TOF timing can reach 10 ps. A detector that does not require light energy conversion would go a long way to achieving this goal. Algorithm developments will continue that correct for attenuation and motion using only TOF information. The reduction in dose will go hand in hand with the improvement in PET technology. For dynamic cardiac PET, it is important to ensure accuracy of the reconstructed data particularly during the high activity period of the blood input phase. The dead time correction and the threshold activity that paralyzes the detector electronics should be verified and measured by the user, preferably also provided by the vendor. Finally, every cardiac PET study should be performed dynamically, even if only a static result is all that is needed; nothing is lost and much can be gained by performing the study dynamically.

\section{Disclosure} disclose.

The authors declare that there is no conflict of interest to

\section{References}

1. Beyer T, Townsend DW, Brun T, Kinahan PE, Charron M, Roddy R, Jerin J, Young J, Byars L, Nutt R. A combined PET/CT scanner for clinical oncology. J Nucl Med. 2000;41:1369-79.

2. http://www.auntminnie.com/index.aspx $? \mathrm{sec}=\sup \&$ sub=mol\&pag= dis\&ItemID $=115325$.

3. Dorbala S, Blankstein R, Skali H, Park MA, Fantony J, Mauceri C, Semer J, Moore SC, Di Carli MF. Approaches to reducing radiation dose from radionuclide myocardial perfusion imaging. J Nucl Med. 2015;56:592-9.

4. Renaud JM, Yip K, Guimond J, Trottier M, Pibarot P, Turcotte E, Maguire C, Lalonde L, Gulenchyn K, Farncombe T, Wisenberg G, Moody J, Lee B, Port SC, Turkington TG, Beanlands RS, deKemp RA. Characterization of 3-dimensional PET systems for accurate quantification of myocardial blood flow. $J$ Nucl Med. 2017;58:103-9.

5. Seo Y, Teo B-K, Hadi M, Schreck C, Bacharach SL, Hasegawa BH. Quantitative accuracy of PET/CT for image-based kinetic analysis. Med Phys. 2008;35:3086-9.

6. Kolthammer JA, Su KH, Grover A, Narayanan M, Jordan DW, Muzic RF. Performance evaluation of the Ingenuity TF PET/CT scanner with a focus on high count-rate conditions. Phys Med Biol. 2014;59:3843-59.

7. Hsu DFC, Ilan E, Peterson WT, Uribe J, Lubberink M, Levin CS. Studies of a next-generation silicon-photomultiplier-based timeof-flight PET/CT system. J Nucl Med. 2017;58:1511-8.

8. Nguyen NC, Vercher-Conejero JL, Sattar A, Miller MA, Maniawski PJ, Jordan DW, Muzic RF Jr, Su KH, O’Donnell JK,
Faulhaber PF. Image quality and diagnostic performance of a digital PET prototype in patients with oncologic diseases: Initial experience and comparison with analog PET. J Nucl Med. 2015;56:1378-85

9. Slomka PJ, Pan T, Germano G. Recent advances and future progress in PET instrumentation. Semin Nucl Med. 2016;46:5-19.

10. Karp JS, Surti S, Daube-Witherspoon ME, Muehllehner G. Benefit of time-of-flight in PET: Experimental and clinical results. J Nucl Med. 2008;49:462-70.

11. Lewellen TK. Time-of-flight PET. Semin Nucl Med. 1998;28:26875 .

12. Roncali E, Cherry SR. Application of silicon photomultipliers to positron emission tomography. Ann Biomed Eng. 2011;39:1358-77.

13. Berg E, Roncali E, Hutchcroft W, Qi J, Cherry SR. Improving depth, energy and timing estimation in PET detectors with deconvolution and maximum likelihood pulse shape discrimination. IEEE Trans Med Imag. 2016;35:2436-46.

14. Lecoq P. Pushing the limits in time-of-flight PET imaging. IEEE Trans Rad Plasma Med Sci. 2017;1:473-85.

15. Gu Y, Matteson JL, Skelton RT, et al. Study of a high-resolution, 3D positioning cadmium zinc telluride detector for PET. Phys Med Biol. 2011;56:1563-84.

16. Berg E, Cherry SR. Using convolutional neural networks to estimate time-of-flight from PET detector waveforms. Phys Med Biol. 2017. https://doi.org/10.1088/1361-6560/aa9dc5 [Epub ahead of print].

17. Qi JY, Leahy RM. Iterative reconstruction techniques in emission computed tomography. Phys Med Biol. 2006;51:R541-78.

18. Leahy RM, Qi J. Statistical approaches in quantitative positron emission tomography. Stat Comput. 2000;10:147-65.

19. Iriarte A, Marabini R, Matej S, Sorzano CO, Lewitt RM. System models for PET statistical iterative reconstruction: A review. Comput Med Imaging Gr. 2016;48:30-48.

20. Brady SL, Shulkin BL. Ultralow dose computed tomography attenuation correction for pediatric PET CT using adaptive statistical iterative reconstruction. Med Phys. 2015;42:558-66.

21. Defrise M, Rezaei A, Nuyts J. Time-of-flight PET data determine the attenuation sinogram up to a constant. Phys Med Biol. 2012;57:885-99.

22. Xu J, Tsui BMW. Improved intrinsic motion detection using timeof-flight PET. IEEE Trans Med Imag. 2015;10:2131-45.

23. Germino M, Carson RE. Cardiac-gated parametric images from ${ }^{82} \mathrm{Rb}$ PET from dynamic frames and direct $4 \mathrm{D}$ reconstruction. Med Phys. 2017. https://doi.org/10.1002/mp.12710 [Epub ahead of print].

24. Ziadi MC. Myocardial flow reserve (MFR) with positron emission tomography (PET)/computed tomography (CT): Clinical impact in diagnosis and prognosis. Cardiovasc Diagn Ther. 2017;72:206-18.

25. Dilsizian V, Taillefer R. Journey in evolution of nuclear cardiology: Will there be another quantum leap with the F-18-labeled myocardial perfusion tracers? JACC Cardiovasc Imaging. 2012;5:1269-84.

26. Hernandez AM, Murphy ST, Zeng GL, Janabi M, Huber JS, Brennan KM, O'Neil JP, Seo Y, Gullberg GT. Longitudinal evaluation of left ventricular substrate metabolism, perfusion, and dysfunction in the SHR model of hypertrophy using microPET imaging. J Nucl Med. 2013;54:1938-45.

27. Katz AM. Physiology of the heart. 5th ed. Philadelphia, PA: Wolters Kluwer Health/Lippincott Williams \& Wilkins Health; 2011.

28. Thackeray JT, Bengel FM. PET imaging of the autonomic nervous system. Q J Nucl Med Mol Imaging. 2016;60:362-82.

29. Boutagy NE, Sinusas AJ. Recent advances and clinical applications of PET cardiac autonomic nervous system imaging. Curr Cardiol Rep. 2017;19:33. 
30. Ketchum ES, Jacobson AF, Caldwell JH, Senior R, Cerqueira MD, Thomas GS, Agostini D, Marula J, Levy WC. Selective improvement in Seattle heart failure model risk stratification using iodine-123 metaiodobenzylguanidine imaging. J Nucl Cardiol. 2012;19:1007-16.

31. Zan Y, Boutchko R, Huang Q, Li B, Chen K, Gullberg GT. Longitudinal evaluation of sympathetic nervous system and perfusion in normal and spontaneously hypertensive rat hearts with dynamic single-photon emission computed tomography. Mol Imaging. 2015;14:373-84.

32. Werner RA, Rischpler C, Onthank D, Lapa C, Robinson S, Samnick S, Javadi M, Schwaiger M, Nekolla SG, Higuchi T. Retention kinetics of the ${ }^{18} \mathrm{~F}$-labeled sympathetic nerve PET tracer LMI1195: Comparison with ${ }^{11} \mathrm{C}-$ Hydroxyephedrine and ${ }^{123} \mathrm{I}$ MIBG. J Nucl Med. 2015;56:1429-33.

33. Mather KJ, DeGrado TR. Imaging of myocardial fatty acid oxidation. Biochim Biophys Acta. 2016;1861:1535-43.

34. de las Fuentes L, Soto PF, Cupps BP, Pasque MK, Herrero P, Gropler RJ, Waggoner AD, Davila-Roman VG. Hypertensive left ventricular hypertrophy is associated with abnormal myocardial fatty acid metabolism and myocardial efficiency. J Nucl Cardiol. 2006;13:369-77.

35. Neely JR, Morgan HE. Relationship between carbohydrate and lipid metabolism and the energy balance of heart muscle. Ann Rev Physiol. 1974;36:413-59.

36. Haim TE, Wang W, Flagg TP, Tones MA, Bahinski A, Numann RE, Nichols CG, Nerbonne JM. Palmitate attenuates myocardial contractility through augmentation of repolarizing $\mathrm{Kv}$ currents. J Mol Cell Cardiol. 2010;48:395-405.

37. Tuunanen H, Engblom E, Naum A, Nagren K, Hesse B, Airaksinen KE, Nuutila P, Iozzo P, Ukkonen H, Opie LH, Knuuti J. Free fatty acid depletion acutely decreases cardiac work and efficiency in cardiomyopathic heart failure. Circulation. 2006;114:2130-7.

38. Wehrl HF, Wiehr S, Divine M, Gatidis S, Gullberg GT, Maier FC, Rolle AM, Schwenk J, Thaiss W, Pichler BJ. Preclinical and translational PET/MR imaging. J Nucl Med. 2014;55:11S-8S. 